\title{
Diseño de un modelo correlacional en la formación del ingeniero industrial como capital humano
}

\author{
Desing of a correlational model in the formation of the industrial engineer as a human \\ capital
}

\author{
Manuel Antonio Leon Julca ${ }^{1}$
}

\begin{abstract}
RESUMEN
Objetivo: Diseñar un modelo de formación profesional del Ingeniero Industrial como capital humano, que disponga de conocimientos actualizados, con habilidades de creatividad e innovación, con capacidades de liderar acciones y una utilización de tecnología de comunicaciones moderna y eficaz. Material y Métodos: El estudio realizado corresponde a una indagación sobre un modelo experimental de formación del profesional de ingeniería industrial con características de capital humano, bajo el esquema de cuatro pilares; los conocimientos, la creatividad e innovación, el liderazgo y la comunicación. Para ello se han tenido en cuenta los estudios sobre el Diagnóstico de la formación actual del ingeniero industrial, los enfoques de distintas universidades nacionales sobre el Perfil del Ingeniero Industrial para el Siglo XXI, la encuesta a egresados y profesionales de ingeniería sobre cómo fue y como debió ser, su formación profesional, y sobre el éxito profesional. La obtención de resultados fue procesado en el SPSS. Resultados: El análisis de la formación profesional del ingeniero industrial comprende dos instantes, el cómo fue y el cómo debió ser. Ante la pregunta de cómo fue la formación profesional, respondieron con una aprobación promedio de casi doce, pero cuando evalúan como debió ser, esta formación profesional, responden con un promedio de diecisiete, obteniéndose una preocupante diferencia. En cuanto al éxito profesional los egresados recomiendan mayor aplicación de los talentos, ser competente, capacitación continua y obtención de experiencias. Conclusiones: Sobre formación profesional, los egresados de Ingeniería Industrial han tenido mayores expectativas y que no se está atendiendo. Sobre el éxito profesional, es vital que el egresado debe desarrollar sus talentos, debe mostrar ser competente, estar en capacitación continua y la experiencia que vienen adquiriendo.
\end{abstract}

Palabras clave: Capital humano, competencias, éxito profesional, modelo de formación profesional, talento humano.

\begin{abstract}
Objective: To design a model of professional training of the Industrial Engineer as human capital, which has updated knowledge, with creativity and innovation skills, with the ability to lead actions and use modern and effective communications technology. Material and Methods: The study carried out corresponds to an inquiry about an experimental model of training of industrial engineering professionals with characteristics of human capital, under the four pillars scheme; knowledge, creativity and innovation, leadership and communication. To this end, the studies on the Diagnosis of the current training of the industrial engineer, the approaches of different national universities on the Profile of the Industrial Engineer for the XXI Century, the survey of graduates and engineering professionals on how it was and how it must have been, his professional training, and professional success. The obtaining of results was processed in the SPSS. Results: The analysis of the professional training of the industrial engineer includes two moments, how it was and how it should have been. When asked how was the professional training, responded with an average approval of almost twelve, but when they evaluate how it should be, this professional training, respond with an average of seventeen, obtaining a worrying difference. In terms of professional success, graduates recommend greater application of talents, be competent, continuous training and obtaining experiences. Conclusions: On professional training, Industrial Engineering graduates have had higher expectations and that is not being attended. On professional success, it is vital that the graduate must develop their talents, must show competence, be in continuous training and the experience they have acquired.
\end{abstract}

Keywords: Human capital, competencies, professional success, professional training model, human talent.

\section{INTRODUCCIÓN}

A través de esta investigación lo que se persigue es proponer una alternativa de formación que conlleve a la buena preparación del ingeniero industrial, con los lineamientos de capital humano; y asimismo evaluando las nuevas capacidades profesionales relacionadas al empleo, señalando las capacidades requeridas por el mercado y estableciendo los factores que influyen en el logro del éxito profesional; como lo exige los tiempos actuales.

Tal como lo sustentan Pere Scorza (2001), y Chavenatto (2002), que las instituciones deben optar por nuevos modelos de formación del capital humano, queda claro entonces, que dentro de los elementos que participan para alcanzar el desarrollo de una institución se encuentra el factor humano que dispone la empresa, el cual puede potenciarse para lograr el desarrollo organizacional. En ese sentido como parte integrante de la Universidad Nacional José Faustino Sánchez Carrión, se justifica realizar estudios sobre el ingeniero industrial que se forma, el mismo que participa en diferentes tipos de organizaciones contenidas en su campo de acción.
Asimismo se ha detectado que a la fecha (2006), la formación profesional del Ingeniero Industrial en la Universidad Nacional José Faustino Sánchez Carrión, así como en otras del sistema universitario, no tiene un modelo de formación actualizado y moderno, afectando a la competitividad de sus profesionales egresados.

Para la buena formación profesional de una persona intervienen una serie de factores, pero generalmente se da mayor énfasis a lo que son los conocimientos. Si hacemos un análisis veremos que la eficiencia y eficacia profesional se ve influida de alguna manera por otros factores.

En consecuencia el objeto de esta investigación es el modelo correlacional del profesional de ingeniería industrial como capital humano de una organización y su actividad de inserción con la eficiencia y eficacia profesional en el campo laboral, donde el Ingeniero Industrial aporta sus capacidades de creación, su experiencia para indicar pautas en diversas áreas, y sus actitudes de gestión en el accionar de actividades en las organizaciones, como son la producción, la administración, el de sistemas e informática y el de proyectos.

\footnotetext{
1 Ingeniero Industrial, Maestro en Docencia Universitaria y Doctor en Ingeniería.

Recibido: 20/11/2018 Aprobado: 15/03/2019
} 
La Historia ha demostrado que la verdad científica, es relativa en el tiempo y se encuentra en evolución constante. Por eso los líderes de la gestión del cambio deben desarrollar una capacidad de flexibilidad, comprensión y persuasión para entender la resistencia y convencer a sus audiencias de la necesidad de innovarse sin emplear la imposición.

Miremos a nuestro alrededor y observemos que la tecnología ha eliminado una serie de trabajos no calificados, mientras que la necesidad de trabajos mediana y altamente calificados va en aumento. La necesidad de tener una visión puesta en el futuro, con un trabajo flexible y un trabajador adaptable e incluso modificable existe; el trabajo real está sustituyendo al trabajo prescrito o tradicional.

Para analizar estas nuevas necesidades del Ingeniero Industrial, se ha tenido en cuenta los aportes que se han vertido en los dos últimos eventos del Consejo Nacional de Facultades y Escuelas de Ingeniería Industrial (CONFINI) celebrados en la ciudad de Lima, el primero realizado en la Universidad Norbert Wiener septiembre 2004, y la siguiente en octubre del mismo año en la Universidad Ricardo Palma con la asistencia de 16 delegaciones del país, y en esta última contando con la participación de seis delegaciones representantes de la Asociación de Carreras de Ingeniería de América Latina y el Caribe (LACCEI ).

\section{MATERIAL Y MÉTODOS}

La metodología que se ha empleado consta de estudios relacionados para llegar a la formulación de una propuesta del modelo siguiendo las siguientes etapas:

a. Diagnóstico de la formación actual del ingeniero industrial. Las instituciones Universitarias y académicas que forman profesionales de esta especialidad, así como el capítulo de Ingenieros Industriales del propio Colegio de Ingenieros del Perú, proporcionan sus criterios sobre este caso.

b. Enfoques sobre el Perfil del Ingeniero Industrial para el Siglo XXI. Instituciones universitarias como la ANR y otras relacionadas a la actividad de Ingeniería Industrial como es el Consejo Nacional de Facultades y Escuelas de Ingeniería Industrial (CONFINI) aglutinan los aportes dados por los representantes de las Universidades que cuentan con esta especialidad.

c. Encuesta a egresados y profesionales de ingeniería sobre el éxito profesional. Los egresados a través de sus experiencias aportan o dan pautas de sus actividades que realizan como ingenieros y de lo que las organizaciones requieren del recurso humano, o en todo caso del capital humano que ellos disponen en sus instituciones.

d. Experimentación del modelo a través de criterios de expertos. Una posición o enfoque de cómo debe ser la formación de un ingeniero industrial de acuerdo a la realidad del nuevo siglo XXI y basándose en un nuevo planteamiento de factores.

e. Encuesta a egresados y profesionales de ingeniería sobre cómo fue y como debió ser, su formación profesional.

Los egresados a través de sus experiencias en el mercado laboral nos dan sus opiniones acerca, de cómo fue y como debió ser su formación laboral, bajo los parámetros de los 4 factores antes citados.

\section{RESULTADOS}

Sobre la formación profesional:

Este punto comprende el análisis de dos instantes, el primero de cómo fue y en segundo lugar como debió ser, la formación profesional del Ingeniero Industrial.

Los resultados que aparecen en la Tabla 1, nos muestra de manera general y bajo una escala vigesimal; ante la pregunta de, cómo fue la formación profesional, respondieron con una aceptación promedio aprobatorio de casi doce (11.97), pero cuando de manera general evalúan como debió ser, esta formación profesional, responden con un promedio de diecisiete $(17,4781)$, obteniéndose una diferencia relacionada entre medias de 5,5054. Estos resultados indican que la tarea de formar profesionales de ingeniería, la estamos cumpliendo bajo un calificativo de regular, lo que nos mueve a pensar y actuar que debemos buscar otras alternativas en pos de revertir esta situación.

Tabla 1

Indicadores generales de la muestra como fue y como debió

\begin{tabular}{|c|c|c|c|c|c|c|c|c|c|}
\hline \multirow[b]{2}{*}{ Descripción } & \multirow[b]{2}{*}{ Media } & \multirow[b]{2}{*}{$\mathbf{S}$} & \multicolumn{2}{|c|}{ Diferencias relacionadas } & \multicolumn{3}{|c|}{ Prueba de medias } & \multicolumn{2}{|c|}{ Decisión } \\
\hline & & & Media & $\mathbf{S}$ & $\mathbf{t}$ & g.l. & $P($ sig.) & Si & No \\
\hline $\begin{array}{l}\text { Evaluación total } \\
\text { Como fue }\end{array}$ & 11,973 & 1,955 & & & & & & Si & \\
\hline $\begin{array}{l}\text { Evaluación total } \\
\text { Como Debió }\end{array}$ & 17,478 & 1,416 & $-5,505$ & 1,777 & $-33,076$ & 113 & 0,000 & & \\
\hline
\end{tabular}

Nota: Datos de los cuestonarios aplicada a los egresados y alumnos de la Escuela Profesional de Ingenieria Industrial.

Los resultados en la Tabla 2, donde se comparan los factores del modelo propuesto, nos muestra que en ninguno de los factores se ha satisfecho las expectativas de los egresados. 
Tabla 2

Comparación de medias entre factores del modelo entre lo que fué y debió ser

\begin{tabular}{|c|c|c|c|c|c|c|c|c|c|c|c|c|c|}
\hline \multirow[b]{2}{*}{$\begin{array}{l}\text { Areas del } \\
\text { Modelo }\end{array}$} & \multirow[b]{2}{*}{ Descripción } & \multirow[b]{2}{*}{ Estadios } & \multirow[b]{2}{*}{$\mathbf{N}$} & \multirow[b]{2}{*}{ Media } & \multirow[b]{2}{*}{$\mathbf{S}$} & \multirow[b]{2}{*}{$\begin{array}{l}\text { Corre- } \\
\text { lación }\end{array}$} & \multicolumn{2}{|c|}{$\begin{array}{l}\text { Diferencias } \\
\text { relacionadas }\end{array}$} & \multicolumn{2}{|c|}{$\begin{array}{c}\text { Prueba de } \\
\text { medias }\end{array}$} & & \multicolumn{2}{|c|}{$\begin{array}{l}\text { Deci- } \\
\text { sión }\end{array}$} \\
\hline & & & & & & & Media & $\begin{array}{l}\text { Des. } \\
\text { Tipica }\end{array}$ & $t$ & gl & $\mathbf{P}$ & $\mathbf{S i}$ & No \\
\hline \multirow{2}{*}{$\begin{array}{l}\text { Conoci- } \\
\text { mientos }\end{array}$} & \multirow{2}{*}{$\begin{array}{l}\text { Promedio de } \\
\text { conocimientos }\end{array}$} & Como fue & 114 & 11,483 & 1,888 & \multirow[b]{2}{*}{0,389} & \multirow[b]{2}{*}{$-6,02 z 3$} & \multirow[b]{2}{*}{1,853} & \multirow[b]{2}{*}{$-34,699$} & \multirow[b]{2}{*}{113} & \multirow[b]{2}{*}{0} & \multirow[b]{2}{*}{ SI } & \\
\hline & & $\begin{array}{l}\text { Como } \\
\text { debió }\end{array}$ & 114 & 17,506 & 1,374 & & & & & & & & \\
\hline \multirow[b]{2}{*}{ Creatividad } & \multirow[b]{2}{*}{$\begin{array}{l}\text { Promedio de } \\
\text { Creatividad }\end{array}$} & Como fue & 114 & 12,211 & 1,993 & \multirow[b]{2}{*}{0,482} & \multirow[b]{2}{*}{$-5,336$} & \multirow[b]{2}{*}{1,821} & \multirow[b]{2}{*}{$-31,291$} & \multirow[b]{2}{*}{113} & \multirow[b]{2}{*}{0} & \multirow[b]{2}{*}{ SI } & \\
\hline & & $\begin{array}{l}\text { Como } \\
\text { debió }\end{array}$ & 114 & 17,546 & 1,474 & & & & & & & & \\
\hline \multirow{2}{*}{ Liderazgo } & \multirow{2}{*}{$\begin{array}{l}\text { Promedio de } \\
\text { Liderazgo }\end{array}$} & Como fue & 114 & 12,565 & 2,212 & \multirow{2}{*}{0,556} & \multirow{2}{*}{$-6,023$} & \multirow{2}{*}{1,857} & \multirow{2}{*}{$-28,746$} & \multirow{2}{*}{113} & \multirow{2}{*}{0} & \multirow{2}{*}{ SI } & \\
\hline & & Como & 114 & 17,564 & 1,494 & & & & & & & & \\
\hline & & Como fue & 114 & 11,633 & 2,378 & & & & & & & & \\
\hline cación & Comunicación & $\begin{array}{l}\text { Como } \\
\text { debió }\end{array}$ & 114 & 17,297 & 1,708 & 0,471 & $-6,023$ & 2,178 & $-27,762$ & 113 & 0 & SI & \\
\hline
\end{tabular}

Nota: Datos de los cuestonarios aplicada a los egresados y alumnos de la Escuela Profesional de Ingenieria Industrial.

Sobre los factores sociodemográficos, entre las respuestas de opinión obtenidos de como fue estos son los resultados. Variable edad: No hay diferencias. Variable sexo, casi no hay diferencia. Variable años de egresado: No hay diferencias, Variable: Si trabaja o no, aquí si hay diferencias. Variable: área donde trabaja, no hay diferencias que generen preocupación

De otro lado los factores sociodemográficos, respecto a como debio ser, estos son los resultados. Variable edad: No hay diferencias. Variable sexo: se nota que la mujer es más crítica. Variable: años de egresado: Si se muestra que hay diferencias, Variable: Si trabaja o no, aquí hay diferencias tenues. Variable: área donde trabaja, no hay diferencias significativas.

Tabla 3

Correlación entre los planes de Formación como debió ser
En cuanto a la correlación Tabla 3, de los planes de formación profesional se obtiene un coeficiente de Pearson, con una relación inversa de $-0,264$, que nos indica que, cuando el encuestado tiene más años de egresado, éste es menos crítico de cómo debió ser la formación, es decir se queja menos sobre lo que recibió para su formación, lo que nos da pautas valederas para afirmar que antes la formación profesional del ingeniero industrial fue mejor que la actual.

\begin{tabular}{|c|c|c|c|c|c|}
\hline \multirow[t]{2}{*}{ Descripción } & \multirow{2}{*}{$\begin{array}{l}\text { Indice de correlación de } \\
\text { Pearson } \\
\begin{array}{c}\text { Correlación de } \\
\text { Pearson }\end{array}\end{array}$} & \multicolumn{2}{|c|}{$\begin{array}{c}\text { Tiempo de egresado } \\
\text { en clases }\end{array}$} & \multicolumn{2}{|c|}{$\begin{array}{c}\text { Evaluación total como } \\
\text { debió }\end{array}$} \\
\hline & & 1 & & $-0,264$ & ** \\
\hline \multirow[t]{2}{*}{ Tiempo de egresado en clases } & Sig. (bilateral) & & & 0,005 & \\
\hline & $\mathrm{N}$ & 114 & & 114 & \\
\hline \multirow{3}{*}{ Evaluación total como debió } & $\begin{array}{l}\text { Correlación de } \\
\text { Pearson }\end{array}$ & $-0,264$ & $* *$ & 1 & \\
\hline & Sig. (bilateral) & 0,005 & & & \\
\hline & $\mathrm{N}$ & 114 & & 114 & \\
\hline
\end{tabular}

Notas: Datos de los cuestonarios aplicada a los egresados y alumnos de la Escuela Profesional de Ingenieria Industrial.

** La correlación es significativa al nivel de 0,01 (bilateral). ( - ) significa correlación inversa -0,264

** Indica que es un índice de correlación inversa muy significativo

\section{Formulación y propuesta del modelo}

Modelación de la investigación.

El modelo que se va describir, parte en primer lugar de la especificación de un sistema real, el mismo que está constituido por la UNJFSC en todo su contexto, la misma que tiene seis facultades, una de ellas es la facultad de Ingeniería; la cual alberga a la Escuela Académica Profesional de Ingeniería Industrial, la que es materia de estudio en la presente investigación.
La Escuela Académica de Ingeniería Industrial con 30 años de creación (14 de Febrero de 1974), es la más antigua que tiene la Facultad de Ingeniería, cuenta a la fecha (Agosto 2004) con 26 promociones y una población de aproximadamente 1250 egresados. Para el desarrollo lectivo y no lectivo cuenta con 37 docentes de los 80 docentes nombrados que tiene la facultad.

Todas las escuelas académicas incluyendo la de Ingeniería industrial, tienen planes curriculares actualizados al año 1999, 
los mismos que están en aplicación desde el año 2000.

Respecto a estudios de postgrado tenemos docentes con grado de Doctor y con grado de Magíster, $35 \%$ son egresados, $26 \%$ de ellos estan cursando estudios de Post-Grado y el resto $(25 \%)$ no han seguido dichos estudios. En resumen solo 1 tiene el grado Doctor y 6 el grado de Maestro.

El modelo a plantear debe partir de algunas premisas como son las siguientes:

1. El participante o egresado recibe conocimientos que en las empresas solo aplica en un bajo porcentaje de lo percibido en la Universidad.

2. Que las capacidades ó habilidades que desarrolla el alumno en el centro de estudios superiores y que luego las aplica como egresado en las empresas resulta ser mínimo o nulas.

3. Que en su mayoría la actitud que muestra un egresado de nuestra Universidad no tiene la suficiente autoconfianza para poder competir en similares condiciones, a pesar que pueda tenerlo.

4. Las aptitudes con que ingresa el estudiante en la formación profesional como ingeniero no son adecuadamente utilizadas ni incentivadas en su verdadera dimensión y poco se genera el desarrollo de otras aptitudes complementarias..

\section{Definición de conceptos para el nuevo modelo}

Para aclarar lo que se desea implementar en el nuevo modelo, debemos conceptuar lo que significa cada uno de los pilares y lo que corresponde a sus variantes contenidas en cada uno de ellos.

Cuando nos referimos al conocimiento, queremos destacar a la acción o efecto de aprender utilizando la inteligencia, los aspectos de la ciencia y del saber. Sus variantes es que sean: - Actualizados - Innovadores - Multidisciplinario.

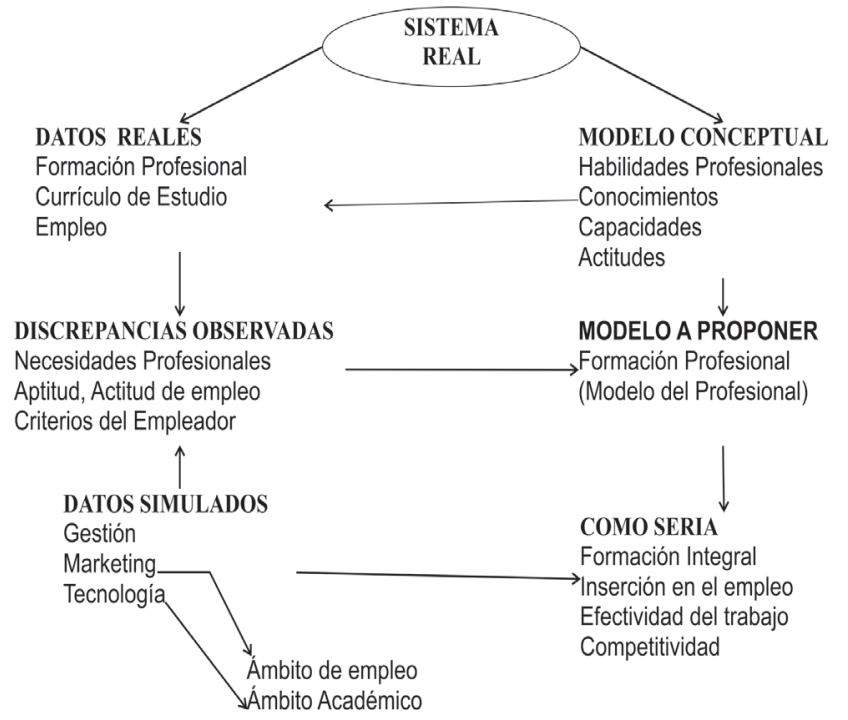

Figura 1: El modelo general del problema.

El Liderazgo son disposiciones naturales o adquiridas para efectuar tareas a través de las personas y con gran eficacia. Es también el culto a las cualidades para el trabajo en equipo de manera estratégica. Las alternativas consideradas para el desarrollo de aptitudes de líder son: -Alto coeficiente de inteligencia -Disposición física para el trabajo en equipo o independiente, -Adaptación a los cambios
El cuarto pilar a considerar es la Comunicación, aspecto importante que en los momentos actuales debemos conocer y utilizar a través de las nuevas tecnologías que están apareciendo. Todo proceso organizacional se rige hacia el futuro por una visión, y en ese sentido se desarrollan a través de la comunicación.

\section{Esquema de desarrollo del modelo.}

En el esquema de desarrollo del modelo, se plantea un nuevo modelo para el Ingeniero industrial, basado en una formación como capital humano que conlleve al desarrollo y uso de los talentos que tiene la persona para hacer más productiva la participación del profesional en el sistema empresarial. (Figura 2):

Se inicia constituyéndose los Fines que persigue el modelo a través de cuatro pilares considerados: conocimientos, creatividad e innovación, liderazgo y comunicación, los mismos que con sus variantes e indicadores consolidan cada uno de estos cimientos en los que se basa el modelo.

Ello conlleva hacia un Proyecto, por un lado de formación científica, tecnológica, humanista y especializada; y por otro lado mediante las actividades de tipo académico, en la práctica profesional, en la investigación y mediante el desarrollo de habilidades para generar la productividad.

Lo que buscamos como Resultado es la inserción de nuestros profesionales en los diversos ámbitos de empleo como el industrial, servicios, finanzas, agroindustria, comercial, pesca, minería, etc. Ver la Figura 2.

Validación del modelo.

La validación se ha efectuado bajo dos criterios:

- Uno es el criterio de expertos, y el otro con,

- La fiabilidad de la escala, mediante el índice de Cronbach.

Mediante el criterio de expertos, el modelo se valida en todos sus factores incluyendo a sus indicadores propuestos, excepto el factor de creatividad e innovación en donde se recomienda un ajuste en la prioridad de sus indicadores, (Gestión empresarial pasa a ocupar la primera prioridad, luego procesos productivos, sistemas y finalmente formular proyectos). De manera similar ocurre en lo que corresponde a los talentos (creador, gestor y técnico) que genera el modelo. El Expert Choice también valida el modelo porque todos los factores obtienen un ratio de inconsistencia menor a 0.10 .

Respecto a la fiabilidad de la escala, a la muestra de egresados se aplicó la consulta de los 4 factores, obteniéndose el índice de Cronbach de 0,8577 lo que nos indica que la muestra tiene muy buena fiabilidad.

\section{DISCUSIÓN}

Con este estudio se demuestra que existe deficiencia en aspectos no cubiertos en la formación profesional del ingeniero industrial de la Universidad Nacional José Faustino Sánchez Carrión.

Los resultados obtenidos también demuestran que todavía existen diferencias entre lo que COMO FUE y lo que DEBIO SER la formación profesional. Esto se concreta cuando se evalúa la propuesta el modelo.

Asimismo es importante el criterio aportado por los egresados respecto a los factores de éxito profesional. Lo que primero destacan es la aplicación del talento, especialmente el 


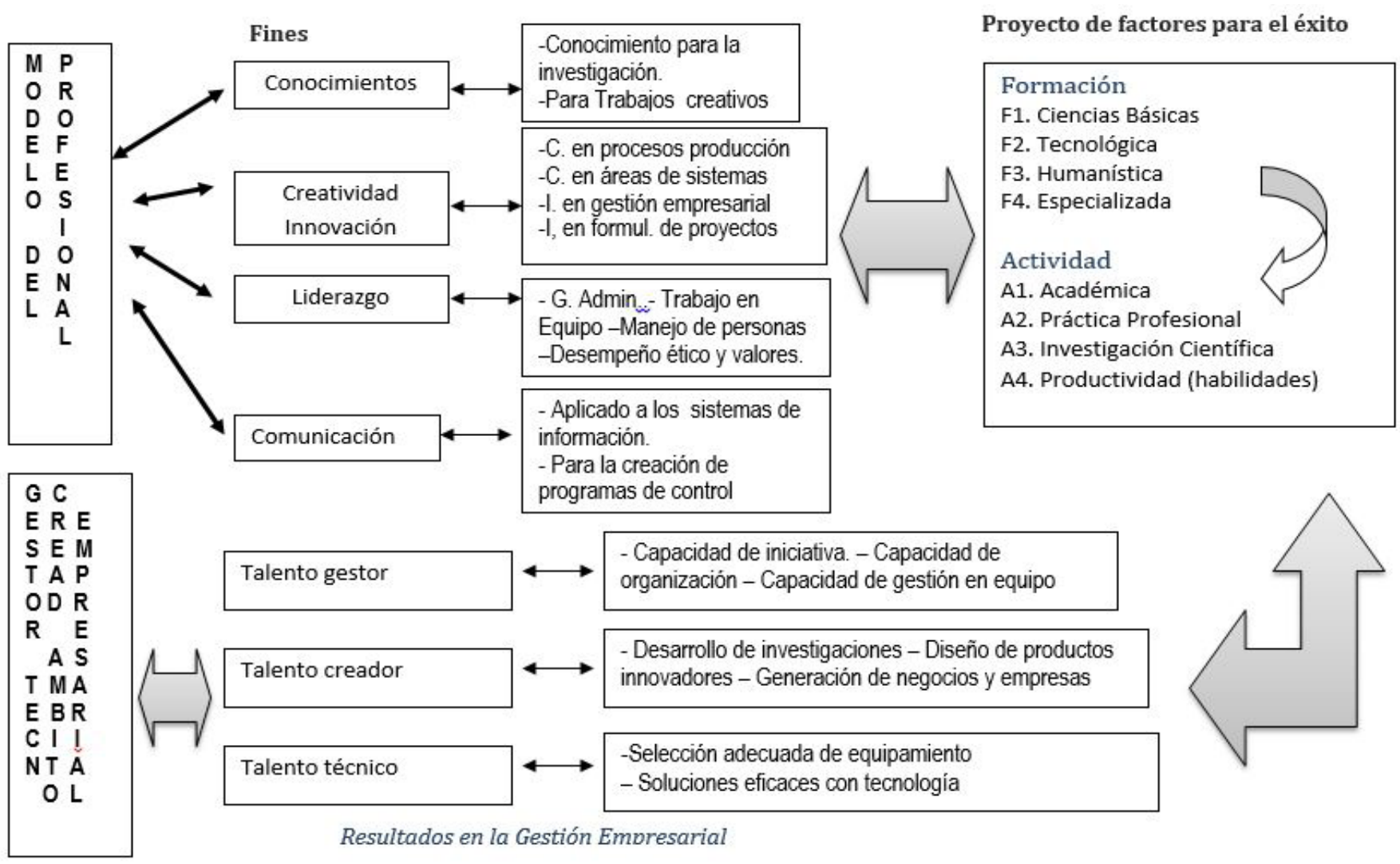

Figura 2: Modelo de formación como capital humano.

creador, y para ser competente el profesional debe tener conocimientos, habilidades, actitud y valores éticos. Destacan de sobre manera la capacitación continua y la experiencia que van adquiriendo complementado con sus habilidades de investigación. Consideran que es un error alcanzar el éxito sacrificando principios éticos o haciendo quedar mal al rival ó recurriendo a ocultar información.

\section{AGRADECIMIENTOS}

A la Universidad del Pacifico por las facilidades brindadas en su Biblioteca para ahondar en los diferentes temas que contiene la presente investigación. Asimismo los grandes aportes sobre sus experiencias, de mis colegas docentes de la Facultad de Ingeniería, y a la valiosa ayuda de mis compañeros de promoción del Doctorado de la Universidad Nacional Federico Villarreal, promoción 2000-2001.

\section{REFERENCIAS BIBLIOGRÁFICAS}

Chiavenato. (2002). Gestión del talento humano. Editorial Mc Graw Hill. México.

Escorsa Castells, Jaume Valls Pasola. (2001). Tecnología e innovación en la empresa dirección y gestión. Ediciones UPC. España. 